Ö. C. Sakinci and J. Beerten, "Impact of PLL dynamics on the small-signal stability of the MMC using dynamic phasor modeling in the ABC frame," Electric Power Systems Research, vol. 189, Dec. 2020, doi: 10.1016/j.epsr.2020.106729.

Digital Object Identifier: https://doi.org/10.1016/j.epsr.2020.106729

URL: https://www.sciencedirect.com/science/article/abs/pii/S0378779620305320 


\title{
Impact of PLL dynamics on the small-signal stability of the MMC using dynamic phasor modeling in the ABC frame ${ }^{\frac{1}{4}}$
}

\author{
Özgür Can Sakinci*, Jef Beerten \\ KU Leuven ESAT/ELECTA \& EnergyVille, Leuven \& Genk, Belgium
}

\section{A R T I C L E I N F O}

\section{Keywords:}

Dynamic phasors

Component connection method

Small-signal modeling

Modular multilevel converter

Phase-locked loop

\begin{abstract}
A B S T R A C T
This paper presents a small-signal dynamic phasor model of the modular multilevel converter including phaselocked loop (PLL) dynamics. A simple PLL model including a low-pass filter at its input and accounting for the harmonic content of the voltage at the point of common coupling is developed to assess the impact of the PLL on small-signal stability. Nonlinear models of the converter, its controllers and the PLL are separately developed and linearized. The linearized models are combined using the component connection method to obtain the overall system matrices. The developed model is verified against a nonlinear averaged model in MATLAB/ Simulink, and dynamic phasor models including different harmonic content are compared in terms of their accuracy. Eigenvalue analysis is carried out to study the impact of the PLL and its filter cutoff frequency on the small-signal stability of the converter.
\end{abstract}

\section{Introduction}

Modular multilevel converters (MMC) are becoming the prominent choice in voltage-source-converter-based high-voltage direct-current (VSC-HVDC) transmission systems, which complicates the analysis of small-signal stability in such systems [1]. Small-signal modeling of the MMC is theoretically challenging as finding a time-invariant operating point to perform linearization in presence of harmonics requires either oversimplifying assumptions or a complicated development process. Dynamic phasor (DP) theory is a promising tool for small-signal modeling of the MMC, as it enables straightforward development of converter models that include the desired harmonic content. Using DP models of the MMC, it has been shown that harmonics above $150 \mathrm{~Hz}$, which are generally neglected in small-signal stability analysis, do result in changes in the damping of critically-damped converter eigenvalues [2].

The traditional $d q$-frame-based control using proportional-integral (PI) controllers is a popular approach in MMC control. In the presence of harmonics, providing the correct angle to perform Park transformations towards $d q$ frames becomes crucial, raising concerns regarding the impact of the phase-locked-loop (PLL) on the dynamic behavior of the converter. The PLL is also known to cause stability issues when the short-circuit ratio (SCR) of the AC grid is low [3]. The impact of the PLL on the stability of VSC-HVDC systems has been studied in [4] with twolevel converters, and in [5] with MMC, demonstrating the significance of detailed converter models in the assessment of the impact of the PLL on small-signal stability. In [6], the PLL dynamics are included in a $d q$ frame-based DP model of the MMC, thereby circumventing the theoretical challenges associated with the DP representation of the Park transformation from the stationary $\mathrm{ABC}$ frame towards $d q$ frames. Moreover, to the authors' knowledge, the small-signal PLL models available in the literature do not consider the harmonic content of the voltage at the point of common coupling (PCC).

This paper builds upon the model introduced in [2], and develops an analytical small-signal model of the MMC including the PLL. The model uses state variables in the stationary $A B C$ frame, and unlike previous applications in the literature, the PLL model developed in this paper makes explicit use of the Park transformation from the stationary $\mathrm{ABC}$ frame towards $d q$ frames and models the interaction of the PLL with the harmonic content present in the PCC voltage. To obtain a realistic PLL representation, the AC-side of the converter is modeled to reflect the changes on the PCC voltage during power transfer. With an accurate representation of the PCC voltage, it becomes theoretically challenging to analytically develop the system equations, as an algebraic loop, depicted in Fig. 1, is inevitably created.

To overcome this challenge, the converter model including the

\footnotetext{
it Research leading to this work was supported by ABB under the ABB research award in honor of Hubertus von Gruenberg. The work of J. Beerten has been supported by the Research Foundation - Flanders (FWO) (Grant No. 12D1117N).

* Corresponding author.

E-mail addresses: ozgurcan.sakinci@esat.kuleuven.be (Ö.C. Sakinci), jef.beerten@esat.kuleuven.be (J. Beerten).
} 


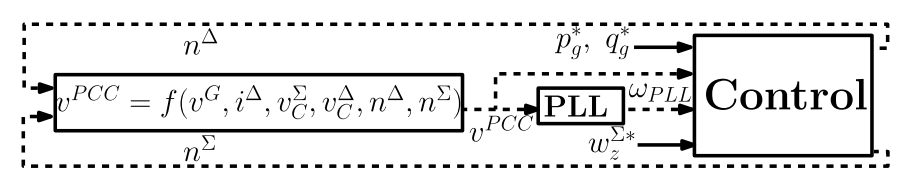

controllers and the PLL is developed by applying the component connection method (CCM). CCM, initially proposed in [7], has mainly been used to combine system matrices of different converters in an interconnected converter system to arrive at the overall system matrices of the interconnected system. In this paper, CCM is used to develop the system matrices of the converter by separately developing the linearized system matrices of its subsystems, i.e. the controllers and the PLL.

The paper first discusses the theory of DPs, and introduces the nonlinear DP model of the MMC which has been developed in detail in [2]. This is followed by the development of the dynamic equations of the controllers. Linearized models of the controllers and the converter are separately calculated, and the overall system matrices are obtained by applying CCM. The developed model is then verified against a nonlinear averaged model in MATLAB/Simulink, by applying smallsignal step tests in time domain. The effect of the PLL on the DP models is investigated by carrying out a participation factor analysis which compares DP models with and without a PLL. Finally, an eigenvalue analysis is carried out for the DP models to analyze the impact of the PLL and the cutoff frequency of the PLL filter on the small-signal stability of the converter.

\section{Dynamic phasor model of the MMC}

In this section, the DP model of the MMC is developed. The theory of DPs is presented first, followed by the introduction of the MMC topology and the nonlinear differential equations guiding the converter dynamics.

\subsection{Theory}

Dynamic phasor theory, initially proposed as a generalized averaging method to improve simulation times of power electronic circuits in [8], is based on the assumption that an arbitrary signal $x(t)$ can be written as a linear superposition of its Fourier coefficients as:

$x(t)=\sum_{k} X_{k} e^{j k \omega_{g} t}$

When transients occur, the dynamic behavior of the signal is approximated by means of time-varying Fourier coefficients:

$x(t)=\sum_{k} X_{k}(t) e^{j k \omega_{g} t}$

The time-varying Fourier coefficients, i.e. dynamic phasors, are obtained by the following moving average:

$X_{k}(t)=\langle x\rangle_{k}(t)=\frac{1}{T} \int_{t-T}^{t} x(\tau) e^{-j k \omega_{g} \tau} d \tau$

In theory, the DP index $k$ is part of an infinite set. In practice, however, it is limited to a bounded, preferably small, set of indices that result in the desired performance. In this paper, the maximum frequency $k \omega_{g}$ that is included in a DP model owing to the maximum index $k$ is hereafter called model bandwidth.

Dynamic phasor modeling, however, can also be a powerful tool in small-signal stability analysis of systems that involve periodicity. A small-signal model that has the DPs as state variables is time invariant by definition as the DPs become constants at steady state. Hence, timeinvariant state-space models can be developed for any set of harmonics in the states and/or inputs by explicitly defining variables for the harmonics inside the model and including their dynamic behavior. In the
Fig. 1. Visualization of the algebraic loop created by the PLL.

development of DP models, the following properties regarding the derivative of a DP and multiplication of two DPs are essential to capture frequency couplings:

$\frac{d\langle x\rangle_{k}}{d t}=\left\langle\frac{d x}{d t}\right\rangle_{k}-j k \omega_{g}\langle x\rangle_{k}$

$\langle x y\rangle_{k}=\sum_{l}\langle x\rangle_{l}\langle y\rangle_{k-l}$

Finally, in a real-valued system, the negative-index DPs are obtained as $\langle x\rangle_{-k}=\langle x\rangle_{k}^{*}$ and the complex DPs are represented as two distinct states corresponding to the real and imaginary parts of $\langle x\rangle_{k}$, i.e. $\langle x\rangle_{k}=\langle x\rangle_{k}^{R}+j\langle x\rangle_{k}^{I}$.

\subsection{Application to the MMC}

The topology of a three-phase MMC including its AC-side representation is given in Fig. 2. The converter model is derived in the stationary $\mathrm{ABC}$ frame, following the methodology described in [2]. A linear transformation from variables in the upper and lower arms towards variables with $\Delta$ and $\Sigma$ superscripts is implemented, which simplifies the frequency content of the state variables [9]. The nonlinear differential equations of the DP model of the MMC for an arbitrary index $k$ are given by:

$$
\begin{aligned}
L_{e q} \frac{d}{d t}\left\langle i_{a b c}^{\Delta}\right\rangle_{k} & =\left\langle v_{a b c}^{\Delta}\right\rangle_{k}-\left\langle v_{a b c}^{G}\right\rangle_{k}-\left(R_{e q}+j k \omega_{g} L_{e q}\right)\left\langle i_{a b c}^{\Delta}\right\rangle_{k} \\
L_{a} \frac{d}{d t}\left\langle i_{a b c}^{\Sigma}\right\rangle_{k} & =\frac{v_{d c}}{2}-\left\langle v_{a b c}^{\Sigma}\right\rangle_{k}-\left(R_{a}+j k \omega_{g} L_{a}\right)\left\langle i_{a b c}^{\Sigma}\right\rangle_{k} \\
\frac{2 C}{N} \frac{d}{d t}\left\langle v_{C a b c}^{\Sigma}\right\rangle_{k} & =\frac{1}{2} \sum_{l=-\infty}^{\infty}\left\langle n_{a b c}^{\Delta}\right\rangle_{k-l}\left\langle i_{a b c}^{\Delta}\right\rangle_{l}+
\end{aligned}
$$

$$
\begin{array}{r}
\sum_{l=-\infty}^{\infty}\left\langle n_{a b c}^{\Sigma}\right\rangle_{k-l}\left\langle i_{a b c}^{\Sigma}\right\rangle_{l}-\frac{2 C}{N} j k \omega_{g}\left\langle v_{C a b c}^{\Sigma}\right\rangle_{k} \\
\frac{2 C}{N} \frac{d}{d t}\left\langle v_{C a b c}^{\Delta}\right\rangle_{k}=\frac{1}{2} \sum_{l=-\infty}^{\infty}\left\langle n_{a b c}^{\Sigma}\right\rangle_{k-l}\left\langle i_{a b c}^{\Delta}\right\rangle_{l}+
\end{array}
$$

$\sum_{l=-\infty}^{\infty}\left\langle n_{a b c}^{\Delta}\right\rangle_{k-l}\left\langle i_{a b c}^{\Sigma}\right\rangle_{l}-\frac{2 C}{N} j k \omega_{g}\left\langle v_{C a b c}^{\Delta}\right\rangle_{k}\left\langle v_{a b c}^{\Sigma}\right\rangle_{k}$

$$
=\frac{1}{2}\left(\sum_{l=-\infty}^{\infty}\left\langle n_{a b c}^{\Sigma}\right\rangle_{k-l}\left\langle v_{C a b c}^{\Sigma}\right\rangle_{l}+\sum_{l=-\infty}^{\infty}\left\langle n_{a b c}^{\Delta}\right\rangle_{k-l}\left\langle v_{C a b c}^{\Delta}\right\rangle_{l}\right)
$$

$\left\langle v_{a b c}^{\Delta}\right\rangle_{k}=-\frac{1}{2}\left(\sum_{l=-\infty}^{\infty}\left\langle n_{a b c}^{\Delta}\right\rangle_{k-l}\left\langle v_{C a b c}^{\Sigma}\right\rangle_{l}+\sum_{l=-\infty}^{\infty}\left\langle n_{a b c}^{\Sigma}\right\rangle_{k-l}\left\langle v_{C a b c}^{\Delta}\right\rangle_{l}\right)$

In (5a), $R_{e q}=R_{G}+R_{T}+R_{a} / 2$ and $L_{e q}=L_{G}+L_{T}+L_{a} / 2$ where $R_{G}$ and $L_{G}$ represent the grid impedance and $R_{T}$ and $L_{T}$ represent the converter transformer. In this paper, two DP models are developed and used in the analysis: one with a model bandwidth of $150 \mathrm{~Hz}$, and another with a model bandwidth of $300 \mathrm{~Hz}$. The models have the DC voltage $\left\langle v_{D C}\right\rangle_{0}$, fundamental frequency and fifth harmonic components of the AC grid voltage $\left\langle v_{a b c}^{G}\right\rangle_{1,-5}$ and the insertion indices $\left\langle n_{a b c}^{\Sigma}\right\rangle_{0,-2,4,6}$ and $\left\langle n_{a b c}^{\Delta}\right\rangle_{1,3,-5}$ as inputs. The state vectors associated with the models are given in (6). 


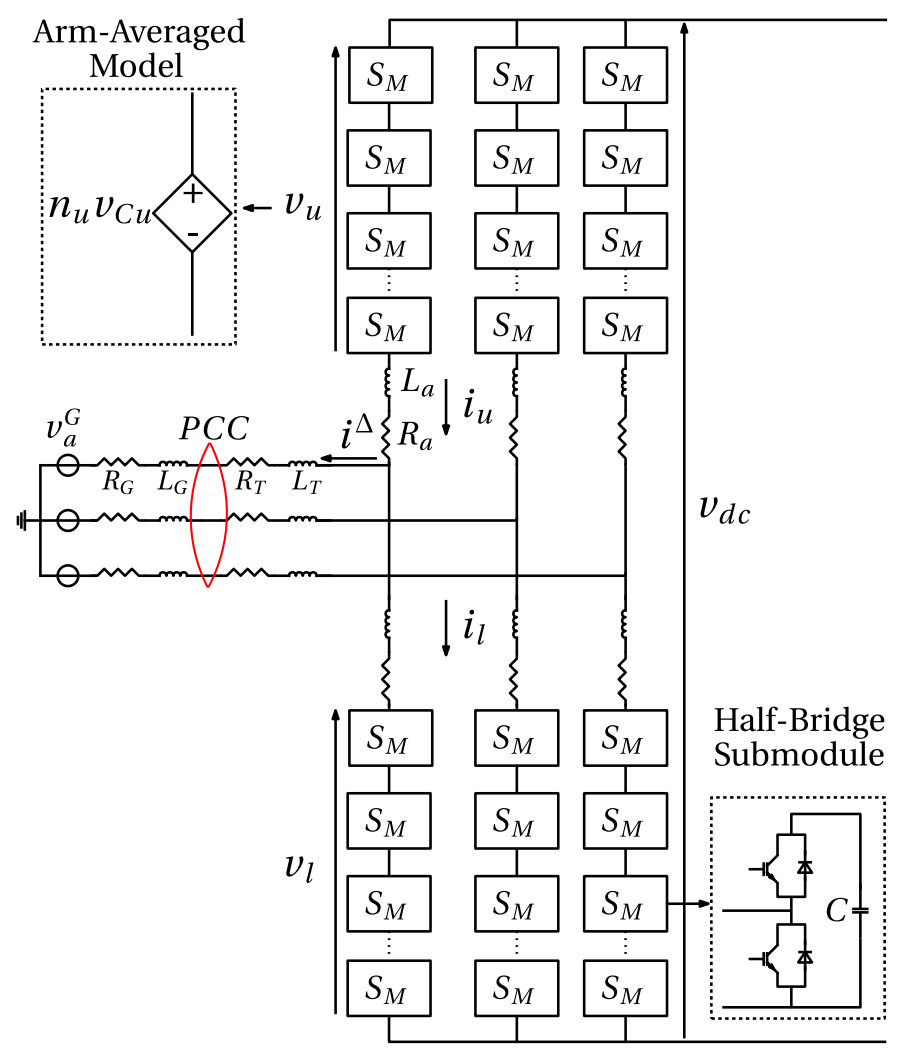

Fig. 2. MMC topology.

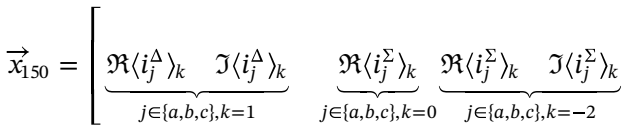

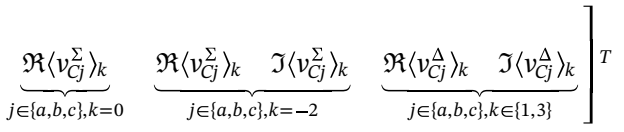

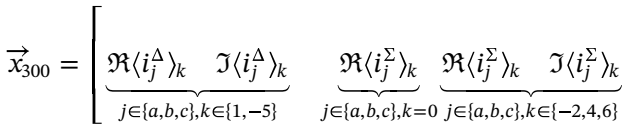

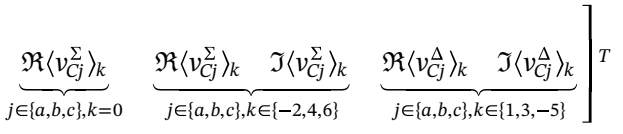

\section{Control architecture}

In this section, the PLL and the controllers included in the DP models are introduced, together with the differential equations guiding their dynamic behavior. When each controller is being introduced, the inputs and outputs of the controller subsystem are also given to help the reader with the application of the CCM and the linerization. An overview of the control architecture, which uses uncompensated modulation to define the insertion indices at the controller outputs [9], is presented in Fig. 3.

\subsection{PLL}

The Park transformations between the stationary ABC frame and an arbitrary $d q$ frame rotating with $n \omega$ is given by:

$$
\begin{aligned}
& \vec{x}_{d q}=\frac{2}{3} \underbrace{\left[\begin{array}{lll}
\cos (n \omega t) & \cos \left(n \omega t-\frac{2 \pi}{3}\right) & \cos \left(n \omega t+\frac{2 \pi}{3}\right) \\
\sin (n \omega t) & \sin \left(n \omega t-\frac{2 \pi}{3}\right) & \sin \left(n \omega t+\frac{2 \pi}{3}\right)
\end{array}\right]}_{T} \vec{x}_{a b c} \\
& \vec{x}_{a b c}=\underbrace{\left[\begin{array}{cc}
\cos (n \omega t) & \sin (n \omega t) \\
\cos \left(n \omega t-\frac{2 \pi}{3}\right) & \sin \left(n \omega t-\frac{2 \pi}{3}\right) \\
\cos \left(n \omega t+\frac{2 \pi}{3}\right) & \sin \left(n \omega t+\frac{2 \pi}{3}\right)
\end{array}\right]}_{T^{-1}} \vec{x}_{d q}
\end{aligned}
$$

When the PLL is set to track the grid frequency $\omega_{g}$, the calculation of the DP equivalents of the transformation matrices $\boldsymbol{T}$ and $\boldsymbol{T}^{-1}$ create a challenge during disturbances in the system frequency. When (3) is applied to the matrices in (7) to calculate the index- $n$ DPs, we obtain:

$\langle\cos (n \omega+\alpha)\rangle_{n}=\left\{\begin{array}{c}0.5(\cos (\alpha)+j \sin (\alpha)), \omega=\omega_{g} \\ 0 \quad, \omega \neq \omega_{g}\end{array}\right.$

which leaves the transformation matrices undefined during transients in the system frequency. Therefore, to avoid such problems, in this paper the PLL is designed to track $\omega_{P L L}$, the deviation from the nominal value of the system frequency $\omega_{g}$. This is equivalent to having a global $d q$ frame that rotates with $\omega_{g}$, and a converter $d q$ frame that rotates with $\omega_{g}+\omega_{P L L}$. The angle that is tracked by the PLL, $\theta_{P L L}$, gives the separation between the two rotating frames. Replacing $n \omega$ with $n \omega_{g}$ in (7) gives the transformation matrices from the $\mathrm{ABC}$ frame towards the global $d q$ frame, and vice versa. In terms of DPs, the Park transformations between the $\mathrm{ABC}$ frame and the global $d q$ frame are given by:

$\left\langle\vec{x}_{d q}^{g}\right\rangle_{0}=\langle\boldsymbol{T}\rangle_{n}\left\langle\vec{x}_{a b c}\right\rangle_{-n}+\langle\boldsymbol{T}\rangle_{-n}\left\langle\vec{x}_{a b c}\right\rangle_{n}$

$\left\langle\vec{x}_{a b c}\right\rangle_{n}=\left\langle\boldsymbol{T}^{-1}\right\rangle_{n}\left\langle\vec{x}_{d q}^{g}\right\rangle_{0}$

With $f_{g}=\omega_{g} / 2 \pi$, the rotations between the global $d q$ frame rotating with $n \omega_{g}$ (denoted with a superscript ' $g$ ') and the converter $d q$ frame rotating with $n\left(\omega_{g}+\omega_{P L L}\right)$ (denoted with a superscript ' $c$ ') are given by 


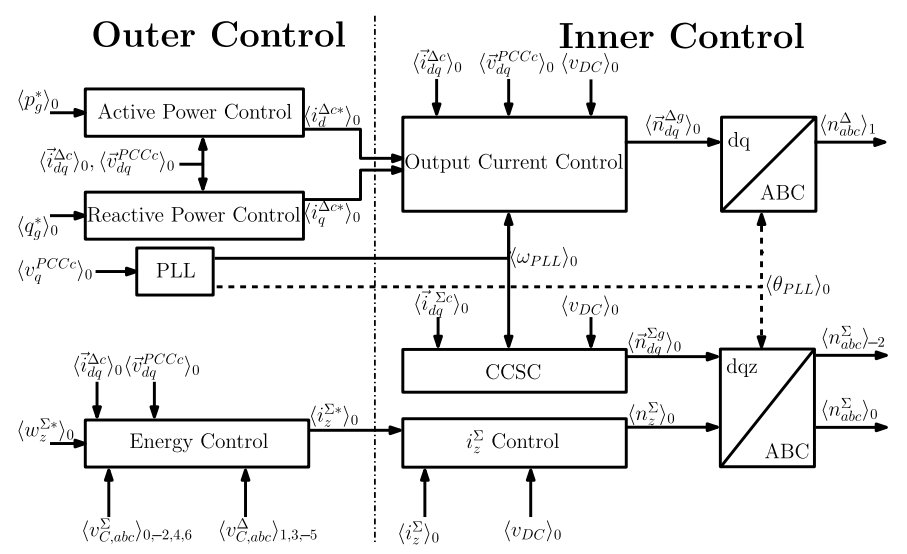

Fig. 3. Controller architecture.

[9]:

$\vec{x}_{d q}^{c}=\boldsymbol{T}_{g-c}^{n f_{g}} \cdot \vec{x}_{d q}^{g}=\left[\begin{array}{cc}\cos \left(n \theta_{P L L}\right) & -\sin \left(n \theta_{P L L}\right) \\ \sin \left(n \theta_{P L L}\right) & \cos \left(n \theta_{P L L}\right)\end{array}\right] \cdot \vec{x}_{d q}^{g}$

$\vec{x}_{d q}^{g}=\boldsymbol{T}_{c-g}^{n f_{g}} \cdot \vec{x}_{d q}^{c}=\left[\begin{array}{cc}\cos \left(n \theta_{P L L}\right) & \sin \left(n \theta_{P L L}\right) \\ -\sin \left(n \theta_{P L L}\right) & \cos \left(n \theta_{P L L}\right)\end{array}\right] \cdot \vec{x}_{d q}^{c}$

The PLL thus tracks the PCC voltage in the converter reference frame to provide the separation angle $\theta_{P L L}$ between the global and the converter reference frames. A low-pass filter with cutoff frequency $f_{P L L}$ is also present, suppressing harmonics in $v_{q}^{P C C c}$ and providing $v_{P L L}$ as a filtered voltage at its output. The dynamic equations of the PLL and the low-pass filter are given by:

$\frac{d\left\langle v_{P L L}\right\rangle_{0}}{d t}=-2 \pi f_{P L L}\left(\left\langle v_{q}^{P C C c}\right\rangle_{0}+\left\langle v_{P L L}\right\rangle_{0}\right)$

$\frac{d\left\langle\epsilon_{P L L}\right\rangle_{0}}{d t}=\left\langle v_{P L L}\right\rangle_{0}$

$\frac{d\left\langle\theta_{P L L}\right\rangle_{0}}{d t}=\left\langle\omega_{P L L}\right\rangle_{0}$

$\left\langle\omega_{P L L}\right\rangle_{0}=K_{P}^{P L L}\left\langle v_{P L L}\right\rangle_{0}+K_{I}^{P L L}\left\langle\epsilon_{P L L}\right\rangle_{0}$

\section{PLL harmonics}

The dynamic equations of the PLL that are given in (11) hold true for the DP model with $150 \mathrm{~Hz}$ bandwidth, yet for the model with $300 \mathrm{~Hz}$ bandwidth, the harmonic content in the PCC voltage, i.e. $\left\langle v_{a b c}^{P C C}\right\rangle_{-5}$ needs to be considered as well. To take the extended harmonic content into account, the PLL model for the DP model with $300 \mathrm{~Hz}$ bandwidth is extended with the states associated with $\left\langle v_{P L L}\right\rangle_{-6},\left\langle\epsilon_{P L L}\right\rangle_{-6}$ and $\left\langle\theta_{P L L}\right\rangle_{-6}$. The 6th harmonic is considered with a negative index in the PLL, because the 5th harmonic at the PCC voltage appears as negative sequence during balanced conditions, i.e. $\left\langle v_{a b c}^{P C C}\right\rangle_{-5}$, and is mapped to $\left\langle v_{q}^{P C C c}\right\rangle_{-6}$ by means of the Park transform at $50 \mathrm{~Hz}$. Similarly, the $4^{\text {th }}$ harmonic circulating current $\left\langle i_{a b c}^{\Sigma}\right\rangle_{4}$ is mapped to $\left\langle i_{d q}^{\Sigma c}\right\rangle_{6}$ by means of the Park transform at $100 \mathrm{~Hz}$. The extended dynamic behavior of the PLL is guided by the following set of equations:

$\frac{d\left\langle v_{P L L}\right\rangle_{-6}}{d t}=-2 \pi f_{P L L}\left(\left\langle v_{q}^{P C C c}\right\rangle_{-6}+\left\langle v_{P L L}\right\rangle_{-6}\right)+j 6 \omega_{g}\left\langle v_{P L L}\right\rangle_{-6}$

$\frac{d\left\langle\epsilon_{P L L}\right\rangle_{-6}}{d t}=\left\langle v_{P L L}\right\rangle_{-6}+j 6 \omega_{g}\left\langle\epsilon_{P L L}\right\rangle_{-6}$

$\frac{d\left\langle\theta_{P L L}\right\rangle_{-6}}{d t}=\left\langle\omega_{P L L}\right\rangle_{-6}+j 6 \omega_{g}\left\langle\theta_{P L L}\right\rangle_{-6}$

$\left\langle\omega_{P L L}\right\rangle_{-6}=K_{P}^{P L L}\left\langle v_{P L L}\right\rangle_{-6}+K_{I}^{P L L}\left\langle\epsilon_{P L L}\right\rangle_{-6}$
Moreover, when the harmonics are taken into consideration, the $6^{\text {th }}$ harmonic components of the rotation matrices in (10) as a result of $\left\langle\theta_{P L L}\right\rangle_{-6}$ also need to be defined. The calculation of $\left\langle\boldsymbol{T}_{g-c}\right\rangle_{-6}$ and $\left\langle\boldsymbol{T}_{c-g}\right\rangle_{-6}$ presents another theoretical challenge, this time associated with the following integral where $\theta_{P L L}$ contains oscillations:

$\left\langle\cos \left(\theta_{P L L}(t)\right)\right\rangle_{-6}(t)=\frac{1}{T} \int_{t-T}^{t} \cos \left(\theta_{P L L}(\tau)\right) e^{j 6 \omega_{g} \tau} d \tau$

To avoid such a theoretical limitation, an approximation has been made regarding the rotation matrices. As $\left\langle\theta_{P L L}\right\rangle_{-6}$ is relatively small, the $6^{\text {th }}$ harmonic components of the rotation matrices can be approximated as follows, replacing the trigonometric functions with quadratic terms that are simpler to represent in terms of DPs:

$\left\langle\boldsymbol{T}_{g-c}^{n f_{g}}\right\rangle_{-6}=\left[\begin{array}{cc}\cos \left(n\left\langle\theta_{P L L}\right\rangle_{-6}\right) & -\sin \left(n\left\langle\theta_{P L L}\right\rangle_{-6}\right) \\ \sin \left(n\left\langle\theta_{P L L}\right\rangle_{-6}\right) & \cos \left(n\left\langle\theta_{P L L}\right\rangle_{-6}\right)\end{array}\right]$

$\approx\left[\begin{array}{cc}-\frac{\left(n\left\langle\theta_{P L L}\right\rangle_{-6}\right)^{2}}{2} & -n\left\langle\theta_{P L L}\right\rangle_{-6} \\ n\left\langle\theta_{P L L}\right\rangle_{-6} & -\frac{\left(n\left\langle\theta_{P L L}\right\rangle_{-6}\right)^{2}}{2}\end{array}\right]$

For the DP model with $300 \mathrm{~Hz}$ bandwidth, the following rotations between the grid and converter references frames are considered for the rest of the controllers:

$\left\langle\vec{i}_{d q}^{\Sigma c}\right\rangle_{0}=\left\langle\boldsymbol{T}_{g-c}^{100}\right\rangle_{0} \cdot\left\langle\vec{i}_{d q}^{\Sigma g}\right\rangle_{0}$

$$
\begin{aligned}
& +\left\langle\mathbf{T}_{g-c}^{100}\right\rangle_{-6} \cdot\left\langle\vec{i}_{d q}^{\Sigma g}\right\rangle_{6}+\left\langle\mathbf{T}_{g-c}^{100}\right\rangle_{6} \cdot\left\langle\vec{i}_{d q}^{\Sigma g}\right\rangle_{-6} \\
& \left\langle\vec{n}_{d q}^{\Sigma g}\right\rangle_{0}=\left\langle\mathbf{T}_{c-g}^{100}\right\rangle_{0} \cdot\left\langle\vec{n}_{d q}^{\Sigma c}\right\rangle_{0}+\left\langle\mathbf{T}_{c-g}^{100}\right\rangle_{-6} \cdot\left\langle\vec{n}_{d q}^{\Sigma c}\right\rangle_{6}+\left\langle\mathbf{T}_{c-g}^{100}\right\rangle_{6} \cdot\left\langle\vec{n}_{d q}^{\Sigma c}\right\rangle_{-6}
\end{aligned}
$$

$\left\langle\vec{n}_{d q}^{\Sigma g}\right\rangle_{6}=\left\langle\mathbf{T}_{c-g}^{100}\right\rangle_{0} \cdot\left\langle\vec{n}_{d q}^{\Sigma c}\right\rangle_{6}+\left\langle\mathbf{T}_{c-g}^{100}\right\rangle_{6} \cdot\left\langle\vec{n}_{d q}^{\Sigma c}\right\rangle_{0}$

$\left\langle\vec{i}_{d q}^{\Delta c *}\right\rangle_{0}=\left\langle\boldsymbol{T}_{g-c}^{50}\right\rangle_{0} \cdot\left\langle\vec{i}_{d q}^{\Delta g *}\right\rangle_{0}$

$\left\langle\vec{i}_{d q}^{\Delta c}\right\rangle_{0}=\left\langle\boldsymbol{T}_{g-c}^{50}\right\rangle_{0} \cdot\left\langle\vec{i}_{d q}^{\Delta g}\right\rangle_{0}$

$+\left\langle\mathbf{T}_{g-c}^{50}\right\rangle_{6} \cdot\left\langle\vec{i}_{d q}^{\Delta g}\right\rangle_{-6}+\left\langle\mathbf{T}_{g-c}^{50}\right\rangle_{-6} \cdot\left\langle\vec{i}_{d q}^{\Delta g}\right\rangle_{6}$

$\left\langle\vec{v}_{d q}^{P C C c}\right\rangle_{0}=\left\langle\mathbf{T}_{g-c}^{50}\right\rangle_{0} \cdot\left\langle\vec{v}_{d q}^{P C C g}\right\rangle_{0}$

$+\left\langle\mathbf{T}_{g-c}^{50}\right\rangle_{6} \cdot\left\langle\vec{v}_{d q}^{P C C g}\right\rangle_{-6}+\left\langle\mathbf{T}_{g_{-c}}^{50}\right\rangle_{-6} \cdot\left\langle\vec{v}_{d q}^{P C C g}\right\rangle_{6}$

$\left\langle\vec{n}_{d q}^{\Delta g}\right\rangle_{0}=\left\langle\mathbf{T}_{c-g}^{50}\right\rangle_{0} \cdot\left\langle\vec{n}_{d q}^{\Delta c}\right\rangle_{0}+\left\langle\mathbf{T}_{c-g}^{50}\right\rangle_{6} \cdot\left\langle\vec{n}_{d q}^{\Delta c}\right\rangle_{-6}+\left\langle\mathbf{T}_{c-g}^{50}\right\rangle_{-6} \cdot\left\langle\vec{n}_{d q}^{\Delta c}\right\rangle_{6}$

$\left\langle\vec{n}_{d q}^{\Delta g}\right\rangle_{-6}=\left\langle\mathbf{T}_{c-g}^{50}\right\rangle_{0} \cdot\left\langle\vec{n}_{d q}^{\Delta c}\right\rangle_{-6}+\left\langle\mathbf{T}_{c-g}^{50}\right\rangle_{-6} \cdot\left\langle\vec{n}_{d q}^{\Delta c}\right\rangle_{0}$ 
The input and output vectors of the PLL subsystem are given by:

$\vec{u}_{P L L}=\left[\left\langle v_{q}^{P C C c}\right\rangle_{0}\left\langle v_{q}^{P C C c}\right\rangle_{-6}\right]^{T}$

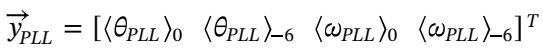

\subsection{PCC voltage}

The voltage at the PCC is calculated using an algebraic equation following the approach in [9]. The PCC voltage in the global $d q$ frame is calculated as:

$\left\langle\vec{v}_{d q}^{P C C g}\right\rangle_{k}=\frac{L_{e q}}{L_{e q}+L_{G}}\left\langle\vec{v}_{d q}^{G g}\right\rangle_{k}+\frac{R_{G} L_{e q}-R_{e q} L_{G}}{L_{e q}+L_{G}}\left\langle\vec{i}_{d q}^{\Delta g}\right\rangle_{k}+\frac{L_{G}}{L_{e q}+L_{G}}\left\langle\vec{v}_{d q}^{\Delta g}\right\rangle_{k}$

\subsection{Outer control layer}

In addition to the PLL, the outer layer control structure includes three PI controllers that achieve active power $p_{g}$, reactive power $q_{g}$ and total energy $w_{z}^{\Sigma}$ tracking. The active power $p_{g}$ and reactive power $q_{g}$ calculated at the PCC are obtained as follows:

$\left\langle p_{g}\right\rangle_{0}=\left\langle v_{d}^{P C C c}\right\rangle_{0}\left\langle i_{d}^{\Delta c}\right\rangle_{0}+\left\langle v_{q}^{P C C c}\right\rangle_{0}\left\langle i_{q}^{\Delta c}\right\rangle_{0}$

$\left\langle q_{g}\right\rangle_{0}=\left\langle v_{q}^{P C C c}\right\rangle_{0}\left\langle i_{d}^{\Delta c}\right\rangle_{0}-\left\langle v_{d}^{P C C c}\right\rangle_{0}\left\langle i_{q}^{\Delta c}\right\rangle_{0}$

The dynamics of the power controllers are guided by states $\left\langle\epsilon_{p}\right\rangle_{0}$ and $\left\langle\epsilon_{q}\right\rangle_{0}$ through:

$\left\langle\dot{\epsilon}_{p}\right\rangle_{0}=\left\langle p_{g}^{*}\right\rangle_{0}-\left\langle p_{g}\right\rangle_{0}$

$\left\langle\dot{\epsilon}_{q}\right\rangle_{0}=\left\langle q_{g}^{*}\right\rangle_{0}-\left\langle q_{g}\right\rangle_{0}$

The controller outputs, namely the references for the output currents $\left\langle\vec{i}_{d q}^{\Delta g *}\right\rangle_{0}$ are given as:

$\left\langle i_{d}^{\Delta g *}\right\rangle_{0}=K_{P}^{P}\left\langle\dot{\epsilon}_{p}\right\rangle_{0}+K_{I}^{P}\left\langle\epsilon_{p}\right\rangle_{0}$

$\left\langle i_{q}^{\Delta g *}\right\rangle_{0}=-\left(K_{P}^{Q}\left\langle\dot{\epsilon}_{q}\right\rangle_{0}+K_{I}^{Q}\left\langle\epsilon_{q}\right\rangle_{0}\right)$

The power control subsystem has the following inputs and outputs (the outputs are obtained by applying (16a)):

$\vec{u}_{p q}=\left[\begin{array}{llll}\left\langle p_{g}^{*}\right\rangle_{0} & \left\langle q_{g}^{*}\right\rangle_{0} & \left\langle\vec{v}_{d q}^{P C C c}\right\rangle_{0} & \left\langle\vec{i}_{d q}^{\Delta c}\right\rangle_{0}\end{array}\right]^{T}$

$\vec{y}_{p q}=\left[\begin{array}{ll}\left\langle\vec{i}_{d q}^{\Delta c *}\right\rangle_{0}\end{array}\right] T$

The final controller in the outer control layer is the energy controller [9]. The total energy stored in the converter is calculated by applying (4b) to the following equation:

$\left\langle w_{z}^{\Sigma}\right\rangle_{0}=\frac{C}{N}\left(\sum_{\substack{j=a, b, c \\ k \in K}}\left\langle v_{C j}^{\Sigma}\right\rangle_{k}^{2}+\sum_{\substack{j=a, b, c \\ k \in K}}\left\langle v_{C j}^{\Delta}\right\rangle_{k}^{2}\right)$

The dynamic behavior of the energy controller is guided by:

$\left\langle\dot{\epsilon}_{w}\right\rangle_{0}=\left\langle w_{z}^{\Sigma *}\right\rangle_{0}-\left\langle w_{z}^{\Sigma}\right\rangle_{0}$
$\left\langle P_{d c}^{*}\right\rangle_{0}=\left\langle p_{g}\right\rangle_{0}+K_{P, w}^{\Sigma}\left\langle\dot{\epsilon}_{w}\right\rangle_{0}+K_{I, w}^{\Sigma}\left\langle\epsilon_{w}\right\rangle_{0}$

The energy controller subsystem has the following inputs and outputs:

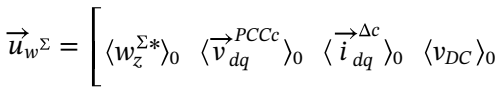

$\left.\left\langle v_{C a b c}^{\Delta}\right\rangle_{1,3,-5}\left\langle v_{C a b c}^{\Sigma}\right\rangle_{0,-2,4,6}\right]^{T}$
$\vec{y}_{w^{\Sigma}}=\left[\left\langle i i_{z}^{\Sigma *}\right\rangle_{0}\right]^{T}$

\subsection{Inner control layer}

The inner control layer consists of the output current control, the circulating current suppression control (CCSC) and the $i_{z}^{\Sigma}$ control. The PI-based output current controller dynamics are given by:

$\left\langle\dot{\epsilon}_{i \vec{d}}\right\rangle_{0}=\left\langle i_{d}^{\Delta c *}\right\rangle_{0}-\left\langle i_{d}^{\Delta c}\right\rangle_{0}$

$\left\langle\dot{\epsilon}_{i} \Delta\right\rangle_{0}=\left\langle i_{q}^{\Delta c *}\right\rangle_{0}-\left\langle i_{q}^{\Delta c}\right\rangle_{0}$

$\left\langle\vec{v}_{d q}^{\Delta c *}\right\rangle_{0}=-\left(\left\langle\vec{v}_{d q}^{P C C c}\right\rangle_{0}+K_{P}^{\Delta}\left\langle\dot{\vec{\epsilon}}_{i_{d q}}\right\rangle_{0}+K_{I}^{\Delta}\left\langle\vec{\epsilon}_{i_{d q}}\right\rangle_{0}\right.$

$$
\begin{aligned}
& +L_{e q} \boldsymbol{J}\left(\omega_{g}+\left\langle\omega_{P L L}\right\rangle_{0}\right)\left\langle\vec{i}_{d q}^{\Delta c}\right\rangle_{0} \\
& \left.+L_{e q} \boldsymbol{J}\left(\left\langle\omega_{P L L}\right\rangle_{6}\left\langle\vec{i}_{d q}^{\Delta c}\right\rangle_{-6}+\left\langle\omega_{P L L}\right\rangle_{-6}\left\langle\vec{i}_{d q}^{\Delta c}\right\rangle_{6}\right)\right) \\
& \left\langle\vec{n}_{d q}^{\Delta c}\right\rangle_{0}=\frac{\left\langle\vec{v}_{d q}^{\Delta c *}\right\rangle_{0}}{\left\langle v_{d c}\right\rangle_{0} / 2} \quad, \quad\left\langle\vec{n}_{d q}^{\Delta c}\right\rangle_{-6}=\left[\begin{array}{ll}
0 & 0
\end{array}\right]^{T}
\end{aligned}
$$

where $\boldsymbol{J}=\left[\begin{array}{cc}0 & 1 \\ -1 & 0\end{array}\right]$. The inputs and outputs of the output current controller subsystem are given by:

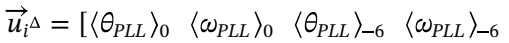

$\left.\left\langle\vec{i}_{d q}^{\Delta c *}\right\rangle_{0}\left\langle\vec{v}_{d q}^{P C C c}\right\rangle_{0}\left\langle\vec{i}_{d q}^{\Delta c}\right\rangle_{0}\left\langle\vec{i}_{d q}^{\Delta c}\right\rangle_{-6}\left\langle v_{D C}\right\rangle_{0}\right]^{T}$

${\overrightarrow{y_{i}}}^{\Delta}=\left[\left\langle n_{a b c}^{\Delta}\right\rangle_{1,-5}\right]^{T}$

The CCSC dynamics are given by:

$\left\langle\dot{\epsilon}_{i} \Sigma\right\rangle_{0}=\left\langle i_{d}^{\Sigma c *}\right\rangle_{0}-\left\langle i_{d}^{\Sigma c}\right\rangle_{0}=-\left\langle i_{d}^{\Sigma c}\right\rangle_{0}$

$\left\langle\dot{\epsilon}_{i_{q}^{\Sigma}}\right\rangle_{0}=\left\langle i_{q}^{\Sigma c *}\right\rangle_{0}-\left\langle i_{q}^{\Sigma c}\right\rangle_{0}=-\left\langle i_{q}^{\Sigma c}\right\rangle_{0}$

$\left\langle\vec{v}_{d q}^{\Sigma c *}\right\rangle_{0}=-K_{P}^{\Sigma}\left\langle\dot{\vec{\epsilon}}_{i d q}\right\rangle_{0}-K_{I}^{\Sigma}\left\langle\vec{\epsilon}_{i_{d q}^{\Sigma}}\right\rangle_{0}$

$+2 L_{a} \boldsymbol{J}\left(\omega_{g}+\left\langle\omega_{P L L}\right\rangle_{0}\right)\left\langle\vec{i}_{d q}^{\Sigma c}\right\rangle_{0}$

$+2 L_{a} \boldsymbol{J}\left(\left\langle\omega_{P L L}\right\rangle_{-6}\left\langle\vec{i}_{d q}^{\Sigma c}\right\rangle_{6}+\left\langle\omega_{P L L}\right\rangle_{6}\left\langle\vec{i}_{d q}^{\Sigma c}\right\rangle_{-6}\right)$

$\left\langle\vec{n}_{d q}^{\Sigma c}\right\rangle_{0}=\frac{\left\langle\vec{v}_{d q}^{\Sigma c *}\right\rangle_{0}}{\left\langle v_{d c}\right\rangle_{0} / 2} \quad, \quad\left\langle\vec{n}_{d q}^{\Sigma c}\right\rangle_{6}=\left[\begin{array}{ll}0 & 0\end{array}\right]^{T}$

The inputs and outputs of the CCSC subsystem are given by:

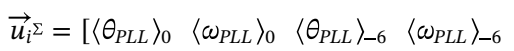

$\left.\left\langle\vec{i}_{d q}^{\Sigma c}\right\rangle_{0}\left\langle\vec{i}_{d q}^{\Sigma c}\right\rangle_{6}\left\langle v_{D C}\right\rangle_{0}\right]^{T}$

${\overrightarrow{y_{i}}}^{\Sigma}=\left[\left\langle n_{a b c}^{\Sigma}\right\rangle_{-2,4}\right]^{T}$

Finally, the $i_{z}^{\Sigma}$ controller dynamics are given by:

$\left\langle\dot{\epsilon}_{i_{z}}^{\Sigma}\right\rangle_{0}=\left\langle i_{z}^{\Sigma *}\right\rangle_{0}-\left\langle i_{z}^{\Sigma}\right\rangle_{0}$

$\left\langle v_{z}^{\Sigma *}\right\rangle_{0}=\frac{\left\langle v_{d c}\right\rangle_{0}}{2}-K_{P z}^{\Sigma}\left\langle\dot{\epsilon}_{i}^{\Sigma}\right\rangle_{0}-K_{I z}^{\Sigma}\left\langle\epsilon_{i z} \Sigma\right\rangle_{0}$

$\left\langle n_{z}^{\Sigma *}\right\rangle_{0}=\frac{\left\langle v_{z}^{\Sigma *}\right\rangle_{0}}{\left\langle v_{d c}\right\rangle_{0} / 2}$

The inputs and outputs of the $i_{z}^{\Sigma}$ controller subsystem are given by: 
$\vec{u}_{i_{z}^{\Sigma}}=\left[\begin{array}{lll}\left\langle i_{z}^{\Sigma *}\right\rangle_{0} & \left\langle i_{z}^{\Sigma}\right\rangle_{0} & \left\langle v_{D C}\right\rangle_{0}\end{array}\right]^{T}$

$\vec{y}_{i_{z}^{\Sigma}}=\left[\left\langle n_{z}^{\Sigma}\right\rangle_{0}\right]^{T}$

The insertion indices at the controller outputs are in different $d q$ frames, but the converter model in (5a) - (5f) has insertion indices in the $\mathrm{ABC}$ frame as inputs. The conversions from the $d q$ frames towards the stationary $\mathrm{ABC}$ frame are implemented by using (9) as:

$\left\langle n_{a b c}^{\Delta}\right\rangle_{1}=\langle\boldsymbol{T}\rangle_{1} \cdot\left\langle\vec{n}_{d q}^{\Delta g}\right\rangle_{0}\left\langle n_{a b c}^{\Delta}\right\rangle_{-5}=\langle\boldsymbol{T}\rangle_{1} \cdot\left\langle\vec{n}_{d q}^{\Delta g}\right\rangle_{-6}$

$\left\langle n_{a b c}^{\Sigma}\right\rangle_{-2}=\langle\boldsymbol{T}\rangle_{-2} \cdot\left\langle\vec{n}_{d q}^{\Sigma g}\right\rangle_{0}\left\langle n_{a b c}^{\Sigma}\right\rangle_{4}=\langle\boldsymbol{T}\rangle_{-2} \cdot\left\langle\vec{n}_{d q}^{\Sigma g}\right\rangle_{6}$

$\left\langle n_{a b c}^{\Sigma}\right\rangle_{0}=\left[\begin{array}{lll}n_{z}^{\Sigma} & n_{z}^{\Sigma} & n_{z}^{\Sigma}\end{array}\right]^{T}$

\section{Model development and validation}

In the previous sections, nonlinear models of the converter and the controllers are derived separately, and their inputs and outputs are defined. In this section, the overall model of the converter and its controllers is defined using CCM. The models defined in the previous sections are considered as subsystems, together with two additional subsystems for $d q$ frame rotations and $v^{P C C}$ calculation, implementing (15), (16) and (18). The subsystems of the overall system are individually linearized by calculating the Jacobian matrices using the state derivatives, states, inputs and outputs associated with each subsystem, as explained in [2]. An overview of the subsystems is depicted in Fig. 4.

The inputs and outputs of the subsystems are combined to form the overall input vector $\vec{u}$ and the overall output vector $\vec{y}$. The output vector of the overall converter subsystem $\vec{b}$ can be chosen as desired, and the input vector $\vec{a}$ is given in (33). Details regarding the application of the CCM are given in Appendix A.

$\vec{a}=\left[\begin{array}{lllll}\left\langle v_{D C}\right\rangle_{0} & \left\langle p_{g}^{*}\right\rangle_{0} & \left\langle q_{g}^{*}\right\rangle_{0} & \left\langle w_{z}^{\Sigma *}\right\rangle_{0} & \left\langle v_{a b c}^{G}\right\rangle_{1,-5}\end{array}\right]^{T}$

DP models with $150 \mathrm{~Hz}$ and $300 \mathrm{~Hz}$ bandwidths are developed, which have 47 and 89 states, respectively. The design parameters of the converter are adapted from [2]. Controller parameters and the AC grid SCR are given in Table 1.

The developed models are verified against a nonlinear averaged model in MATLAB/Simulink for a balanced steady-state operating point with $\left\langle w_{z}^{\Sigma *}\right\rangle_{0}=\left\langle p_{g}^{*}\right\rangle_{0}=\left\langle v_{D C}\right\rangle_{0}=\left|v_{a b c}^{G}\right|=1 \mathrm{pu}$ and $\left\langle q_{g}^{*}\right\rangle_{0}=0 \mathrm{pu}$. The phase A circulating current $i_{a}^{\Sigma}$ responses during a 1\% step change in the fundamental frequency grid voltage $\left\langle v^{G}\right\rangle_{1}$ magnitude and during a $2 \%$ step change in the active power reference $\left\langle p_{g}^{*}\right\rangle_{0}$ are presented in Fig. 5 and Fig. 6, respectively. It can be clearly seen that the DP model with $150 \mathrm{~Hz}$ bandwidth fails to capture the dynamic behavior of the nonlinear model, whereas the DP model with $300 \mathrm{~Hz}$ bandwidth can accurately represent the dynamic behavior of the converter during both step tests.
Table 1

Controller parameters

\begin{tabular}{llllllll}
\hline Symbol & Value & Symbol & Value & Symbol & Value & Symbol & Value \\
\hline$K_{P}^{P}$ & $0.1 \mathrm{pu}$ & $K_{I}^{P}$ & $50 \mathrm{pu}$ & $K_{P}^{Q}$ & $0.1 \mathrm{pu}$ & $K_{I}^{Q}$ & $50 \mathrm{pu}$ \\
$K_{P, w}^{\Sigma}$ & $3.68 \mathrm{pu}$ & $K_{I, w}^{\Sigma}$ & $12.26 \mathrm{pu}$ & $K_{P z}^{\Sigma}$ & $0.05 \mathrm{pu}$ & $K_{I z}^{\Sigma}$ & $10.99 \mathrm{pu}$ \\
$K_{P}^{\Delta}$ & $1.06 \mathrm{pu}$ & $K_{I}^{\Delta}$ & $766.49 \mathrm{pu}$ & $K_{P}^{\Sigma}$ & $0.05 \mathrm{pu}$ & $K_{I}^{\Sigma}$ & $10.99 \mathrm{pu}$ \\
$K_{P}^{P L L}$ & $0.52 \mathrm{pu}$ & $K_{I}^{P L L}$ & $34.62 \mathrm{pu}$ & $f_{P L L}$ & $50 \mathrm{~Hz}$ & SCR & 10 \\
\hline
\end{tabular}

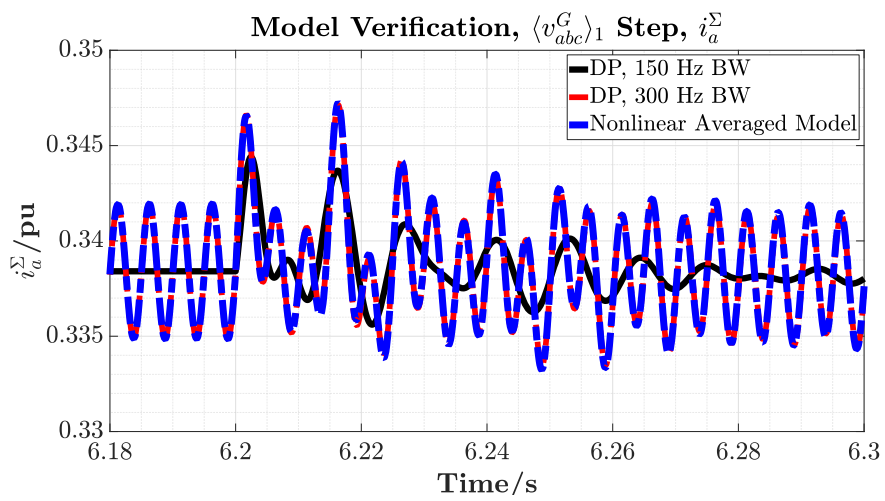

Fig. 5. Comparison of $i_{a}^{\Sigma}$ dynamic responses, $\left\langle v^{G}\right\rangle_{1}$ step.

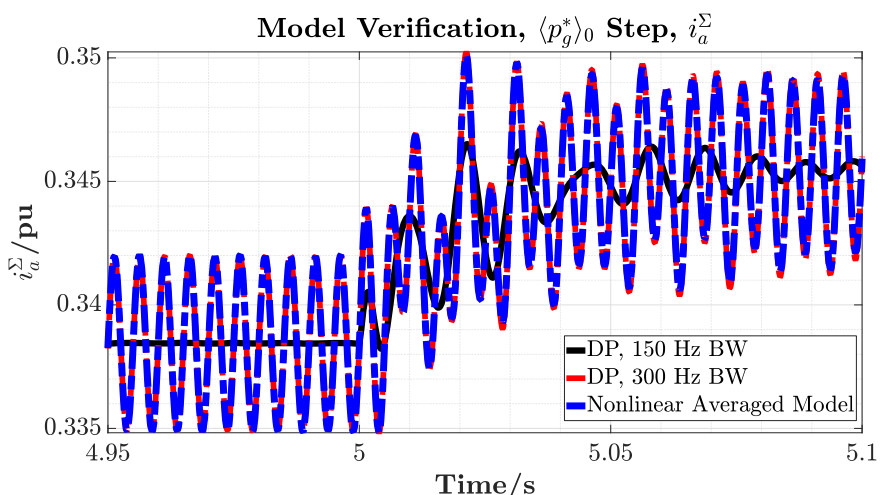

Fig. 6. Comparison of $i_{a}^{\Sigma}$ dynamic responses, $\left\langle p^{g^{*}}\right\rangle_{0}$ step.

\section{Analysis}

To analyze the effect of the PLL on the small-signal stability of the MMC, the eigenvalues of the linearized converter models are calculated for an operating point with a relatively weak AC grid, i.e. $S C R=2$. In the comparison, a variant of the DP model with $300 \mathrm{~Hz}$ bandwidth without the PLL is also used, which uses the same controllers as the other models except the PLL, and assumes all converter variables to lie in the global $d q$ frame. The comparison of the eigenvalues is depicted in

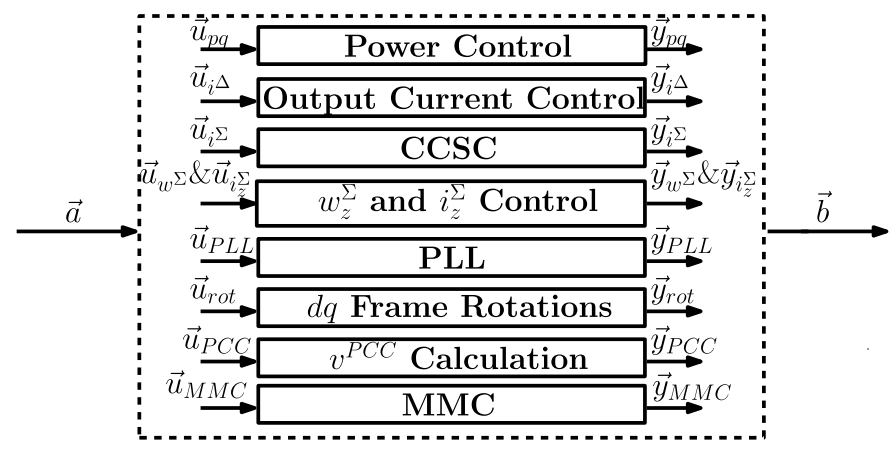

Fig. 4. Overview of subsystems used in the application of CCM. 


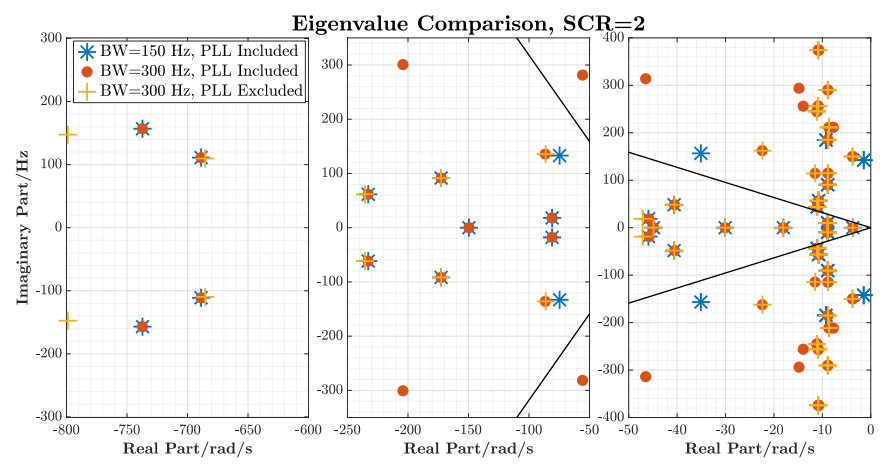

Fig. 7. Eigenvalue comparison.

Table 2

Participation factor comparison.

\begin{tabular}{|c|c|c|}
\hline Eigenvalues & Model Type & Main Participating States \\
\hline$-798.9 \pm 147.52 j$ & $300 \mathrm{~Hz}$ Without PLL & $\left\langle\epsilon_{i \Delta}\right\rangle_{0},\left\langle i_{a b c}^{\Delta}\right\rangle_{1},\left\langle\epsilon_{i} \Delta\right\rangle_{0},\left\langle\epsilon_{q}\right\rangle_{0}$ \\
\hline$-737.2 \pm 156.92 j$ & $300 \mathrm{~Hz}$ With PLL & $\left\langle\epsilon_{i} \Delta\right\rangle_{0},\left\langle i i_{a b c}\right\rangle_{1},\left\langle\epsilon_{q}\right\rangle_{0},\left\langle v_{P L L}\right\rangle_{0},\left\langle\epsilon_{i d} \Delta\right\rangle_{0}$ \\
\hline$-685.39 \pm 109.70 j$ & $300 \mathrm{~Hz}$ Without PLL & $\left\langle\epsilon_{i \Delta}\right\rangle_{0},\left\langle i_{a b c}^{\Delta}\right\rangle_{1},\left\langle\epsilon_{i_{q} \Delta}\right\rangle_{0},\left\langle\epsilon_{p}\right\rangle_{0}$ \\
\hline$-688.78 \pm 111.16 j$ & $300 \mathrm{~Hz}$ With PLL & $\left\langle\epsilon_{i} \Delta\right\rangle_{0},\left\langle i i_{a b c}\right\rangle_{1},\left\langle\epsilon_{p}\right\rangle_{0},\left\langle\epsilon_{i} \Delta\right\rangle_{0},\left\langle v_{P L L}\right\rangle_{0}$ \\
\hline$-47.13 \pm 18.67 j$ & 300 Hz Without PLL & $\left\langle\epsilon_{i} \Sigma\right\rangle_{d},\left\langle\epsilon_{i} \Sigma\right\rangle_{q},\left\langle i_{a b c}^{\Sigma}\right\rangle_{-2},\left\langle v_{C a b c}^{\Sigma}\right\rangle_{-2}$ \\
\hline$-45.81 \pm 18.59 j$ & $300 \mathrm{~Hz}$ With PLL & $\left\langle\epsilon_{i}^{\Sigma}\right\rangle_{0},\left\langle\epsilon_{i}\right\rangle_{d},\left\langle\theta_{P L L}\right\rangle_{0},\left\langle i_{a b c}^{\Sigma}\right\rangle_{-2},\left\langle v_{C a b c}^{\Sigma}\right\rangle_{-2}$ \\
\hline$-8.92 \pm 290.20 j$ & $300 \mathrm{~Hz}$ Without PLL & $\left\langle i_{a b c}^{\Sigma}\right\rangle_{4},\left\langle v_{C a b c}^{\Delta}\right\rangle_{-5}$ \\
\hline$-14.82 \pm 293.81 j$ & $300 \mathrm{~Hz}$ With PLL & $\left\langle\theta_{P L L}\right\rangle_{-6},\left\langle\epsilon_{P L L}\right\rangle_{-6},\left\langle v_{C a b c}^{\Delta}\right\rangle_{-5}$ \\
\hline$-235.46 \pm 61.61 j$ & $300 \mathrm{~Hz}$ Without PLL & $\left\langle i_{a b c}^{\Sigma}\right\rangle_{-2},\left\langle\epsilon_{i}^{\Sigma}\right\rangle_{0},\left\langle\epsilon_{i} \Sigma\right\rangle_{0},\left\langle v_{C a b c}^{\Sigma}\right\rangle_{-2}$ \\
\hline$-232.97 \pm 61.59 j$ & $300 \mathrm{~Hz}$ With PLL & $\left\langle i_{a b c}^{\Sigma}\right\rangle_{-2},\left\langle\epsilon_{i}^{\Sigma}\right\rangle_{0},\left\langle\epsilon_{i q}^{\Sigma}\right\rangle_{0},\left\langle v_{C a b c}^{\Sigma}\right\rangle_{-2}$ \\
\hline$-10.93 \pm 256.28 j$ & $300 \mathrm{~Hz}$ Without PLL & $\left\langle i_{a b c}^{\Delta}\right\rangle_{-5},\left\langle v_{C a b c}^{\Sigma}\right\rangle_{4}$ \\
\hline$-13.94 \pm 256.07 j$ & $300 \mathrm{~Hz}$ With PLL & $\left\langle i_{a b c}^{\Delta}\right\rangle_{-5},\left\langle v_{C a b c}^{\Sigma}\right\rangle_{4}$ \\
\hline$-46.49 \pm 313.90 j$ & $300 \mathrm{~Hz}$ With PLL & $\left\langle\theta_{P L L}\right\rangle_{-6},\left\langle\varepsilon_{P L L}\right\rangle_{-6},\left\langle v_{P L L}\right\rangle_{-6},\left\langle v_{C a b c}^{\Delta}\right\rangle_{-5}$ \\
\hline$-204.17 \pm 300.70 j$ & $300 \mathrm{~Hz}$ With PLL & $\left\langle v_{P L L}\right\rangle_{-6},\left\langle\theta_{P L L}\right\rangle_{-6},\left\langle\epsilon_{P L L}\right\rangle_{-6},\left\langle i \dot{a}_{a b c}^{\Delta}\right\rangle_{-5}$ \\
\hline$-55.74 \pm 281.47 j$ & $300 \mathrm{~Hz}$ With PLL & $\left\langle\theta_{P L L}\right\rangle_{-6},\left\langle\epsilon_{P L L}\right\rangle_{-6},\left\langle v_{P L L}\right\rangle_{-6},\left\langle i_{a b c}^{\sum}\right\rangle_{4}$ \\
\hline-149.66 & $300 \mathrm{~Hz}$ With PLL & $\left\langle\theta_{P L L}\right\rangle_{0},\left\langle v_{P L L}\right\rangle_{0},\left\langle\epsilon_{P L L}\right\rangle_{0},\left\langle\varepsilon_{i} \Delta\right\rangle_{0},\left\langle\epsilon_{i q} \Delta\right\rangle_{0},\left\langle\varepsilon_{p}\right\rangle_{0},\left\langle\varepsilon_{i} \Sigma\right\rangle_{0},\left\langle\epsilon_{i q}^{\Sigma}\right\rangle_{0}$ \\
\hline$-80.93 \pm 17.92 j$ & $300 \mathrm{~Hz}$ With PLL & $\left.\left\langle\theta_{P L L}\right\rangle_{0},\left\langle v_{P L L}\right\rangle_{0},\left\langle\epsilon_{P L L}\right\rangle_{0},\left\langle\epsilon_{i \vec{d}}\right\rangle_{0},\left\langle\epsilon_{i q}\right\rangle_{0}\right\rangle_{0},\left\langle\epsilon_{i \vec{d}}\right\rangle_{0}$ \\
\hline
\end{tabular}

Fig. 7 , in which black lines indicating $5 \%$ damping are also included. It is evident that there are differences in the eigenvalues of different DP models, and new eigenvalues with a damping less than $5 \%$ are observed when the PLL is included in the model. The differences between DP models with $150 \mathrm{~Hz}$ and $300 \mathrm{~Hz}$ bandwidths are in line with the differences reported in [2] for similar DP models excluding the PLL. Therefore, the main focus of this analysis is on the differences due to the inclusion of the PLL in the DP model with $300 \mathrm{~Hz}$ bandwidth. Modal analysis is carried out to better comprehend the reasons behind the changes in the eigenvalues, and the most participating states (ordered from most participating to least participating) associated with the eigenvalues that show considerable changes are presented in Table 2. In Table 2, eigenvalues that shift with the inclusion of the PLL are grouped together with their new values, and for complex-valued DPs, the participation of states corresponding to the real and imaginary parts of the DP are combined. The complex parts of the eigenvalues are given in $\mathrm{Hz}$.

The effect of the PLL can be observed in several complex-conjugate eigenvalue pairs. For the pairs $-235.46 \pm 61.61 j$ and $-10.93 \pm 256.28 j$, the inclusion of the PLL results in minor changes in the damping of the eigenvalues, yet the states participating in the eigenvalue remain unaltered. For the pairs $-798.9 \pm 147.52 j, \quad-685.39 \pm 109.70 j$, $-47.13 \pm 18.67 j$ and $-8.92 \pm 290.20 j$, however, in addition to slight changes in damping, states associated with the PLL start having significant participations in the eigenvalues with the inclusion of the PLL.
Furthermore, when the PLL is included, new modes appear in the model such as -149.66 and $-80.93 \pm 17.92 j$ which are associated with the DCvalued PLL states and the rest of the controllers, and $-46.49 \pm 313.90 j$, $-204.17 \pm 300.70 j$ and $-55.74 \pm 281.47 j$ which are linked to the 6 th harmonic PLL states and the converter voltage and current harmonics.

The DP model with $300 \mathrm{~Hz}$ bandwidth is further used to assess the impact of the cutoff frequency $f_{P L L}$ of the PLL filter. To this end, $f_{P L L}$ is varied, and the changes in the converter eigenvalues are presented in Fig. 8. The directions along which the eigenvalues move as $f_{P L L}$ increases are depicted with solid lines. During variations in $f_{P L L}$, it is observed that even though the major change occurs for eigenvalues that are directly associated with the PLL filter, i.e. -149.66 and $-204.17 \pm 300.70 j$, eigenvalues that have damping less than $5 \%$ and frequency between 200 and $300 \mathrm{~Hz}$ also depend on the PLL filter cutoff frequency.

The results of the analyses carried out in this section reveal that the PLL has a non-negligible impact on the small-signal stability of the MMC, which is observed on both high and low-frequency modes. When the PLL is included in the DP model with $300 \mathrm{~Hz}$ bandwidth, some of the high-frequency eigenvalues associated with the converter voltage and current harmonics show an increase in damping, and as observed in Table 2, states associated with the PLL start having a significant participation in these eigenvalues. 


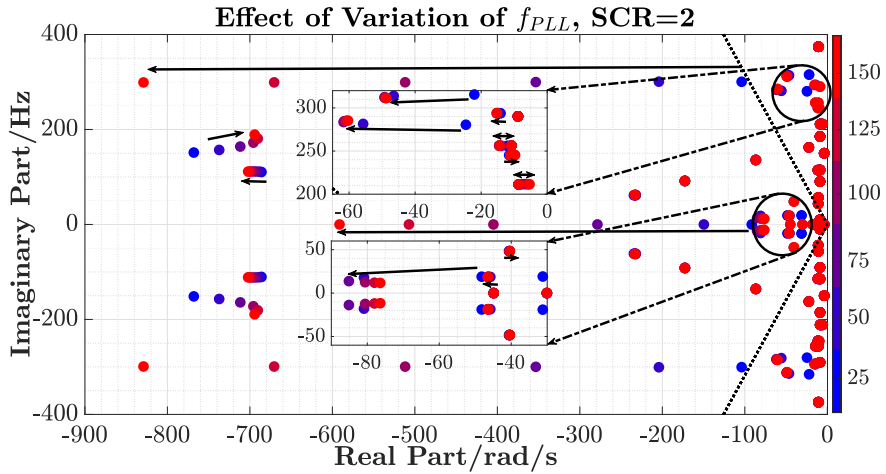

Fig. 8. Eigenvalue changes during $f_{P L L}$ variation.

\section{Conclusions}

This paper presented a small-signal DP model of the MMC including a detailed representation of the PLL to evaluate its impact on the smallsignal stability of the converter. The PLL model is developed considering its interactions with the harmonic content of the PCC voltage. To avoid algebraic loops in the development of the analytical model, linearized models of the controllers, the PLL and the converter are developed separately, and the CCM is applied to obtain the overall system matrices. DP models with $150 \mathrm{~Hz}$ and $300 \mathrm{~Hz}$ bandwidth are developed, and validated in time domain through comparisons with a nonlinear averaged model of the MMC in MATLAB/Simulink. During model validation, it was observed that the DP model with $150 \mathrm{~Hz}$ bandwidth fails to capture the dynamic behavior of the circulating current $i_{a b c}^{\Sigma}$. To assess the impact of the PLL on small-signal stability, eigenvalue analysis is conducted for a relatively weak AC grid with $\mathrm{SCR}=2$ and participation factors of eigenvalues showing major changes when the PLL is included into the model are presented. It was found out that with the inclusion of the PLL in the DP model with $300 \mathrm{~Hz}$ bandwidth, new modes with damping less than $5 \%$ are present, and these modes are mainly associated with the PLL states and converter voltage and current harmonics such as $\left\langle i_{a b c}^{\Sigma}\right\rangle_{4}$ and $\left\langle v_{\text {Cabc }}^{\Delta}\right\rangle_{-5}$. These results show that the inclusion of the PLL in a DP model considering high-frequency harmonics reveals potential couplings between converter harmonics and the PLL, thereby increasing the accuracy of the model.

\section{Declaration of Competing Interest}

The authors declare that they have no known competing financial interests or personal relationships that could have appeared to influence the work reported in this paper.

\section{Appendix A. Component connection method}

Assume a system is composed of several subsystems, where each subsystem has the following generic state-space form:

$\dot{\overrightarrow{x_{i}}}=\boldsymbol{A}_{i} \vec{x}_{i}+\boldsymbol{B}_{i} \overrightarrow{u_{i}}$

$\overrightarrow{y_{i}}=\boldsymbol{C}_{i} \vec{x}_{i}+\boldsymbol{D}_{i} \vec{u}_{i}$

The overall system with state vector $\vec{x}=\left[\vec{x}_{1} \vec{x}_{2} \ldots \vec{x}_{N}\right]^{T}$, input vector $\vec{a}$ and output vector $\vec{b}$ is represented as:

$\dot{\vec{x}}=\boldsymbol{F} \vec{x}+\boldsymbol{G} \vec{a}$

$\vec{b}=\boldsymbol{H} \vec{x}+\boldsymbol{J} \vec{a}$

The interconnection of the subsystems is defined in terms of the interconnection matrices $L_{1: 4}$, the combined input vector $\vec{u}=\left[\vec{u}_{1} \vec{u}_{2} \ldots \vec{u}_{N}\right]^{T}$ and the combined output vector $\vec{y}=\left[\vec{y}_{1} \vec{y}_{2} \ldots \vec{y}_{N}\right]^{T}$ as:

$\vec{u}=L_{1} \vec{y}+L_{2} \vec{a}$

$\vec{b}=L_{3} \vec{y}+L_{4} \vec{a}$

Finally, the overall system matrices are obtained by:

$$
\begin{aligned}
\boldsymbol{F} & =\overline{\boldsymbol{A}}+\bar{B} \boldsymbol{L}_{1}\left(\boldsymbol{I}-\overline{\mathbf{D}} \boldsymbol{L}_{1}\right)^{-1} \overline{\boldsymbol{C}} \\
\boldsymbol{G} & =\bar{B} \boldsymbol{L}_{1}\left(\boldsymbol{I}-\bar{D} \boldsymbol{L}_{1}\right)^{-1} \bar{D} \boldsymbol{L}_{2}+\bar{B} \boldsymbol{L}_{2} \\
\boldsymbol{H} & =\boldsymbol{L}_{3}\left(\boldsymbol{I}-\overline{\mathbf{D}} \boldsymbol{L}_{1}\right)^{-1} \overline{\boldsymbol{C}} \\
\boldsymbol{J} & =\boldsymbol{L}_{3}\left(\boldsymbol{I}-\overline{\mathbf{D}} \boldsymbol{L}_{1}\right)^{-1} \bar{D} \boldsymbol{L}_{2}+\boldsymbol{L}_{4}
\end{aligned}
$$

where $\overline{\boldsymbol{A}}, \overline{\boldsymbol{B}}, \overline{\boldsymbol{C}}, \overline{\boldsymbol{D}}$ are block-diagonal matrices constructed using the system matrices of the subsystems.

\section{References}

[1] A. Antonopoulos, L. Angquist, H.P. Nee, On dynamics and voltage control of the modular multilevel converter, Proc. 2009 13th European Conference on Power Electronics and Applications, (2009). 10 pages

[2] Ö.C. Sakinci, J. Beerten, Generalized dynamic phasor modeling of the MMC for small-signal stability analysis, IEEE Trans. Power Delivery 34 (3) (2019) 991-1000.

[3] T. Midtsund, J.A. Suul, T. Undeland, Evaluation of current controller performance and stability for voltage source converters connected to a weak grid, Proc. The 2nd International Symposium on Power Electronics for Distributed Generation Systems, (2010), pp. 382-388.

[4] J.Z. Zhou, H. Ding, S. Fan, Y. Zhang, A.M. Gole, Impact of short-circuit ratio and phase-locked-loop parameters on the small-signal behavior of a VSC-HVDC converter, IEEE Trans. Power Delivery 29 (5) (2014) 2287-2296.
[5] S. Arunprasanth, R. Kuffel, U.D. Annakkage, C. Karawita, Dynamic behaviour of VSCHVDC systems under different AC system strengths, Proc. 2018 IEEE/PES Transmission and Distribution Conference and Exposition (T D), (2018).

[6] T. Li, A.M. Gole, C. Zhao, Harmonic instability in MMC-HVDC converters resulting from internal dynamics, IEEE Trans. Power Delivery 31 (4) (2016) 1738-1747.

[7] G. Gaba, S. Lefebvre, D. Mukhedkar, Comparative analysis and study of the dynamic stability of AC/DC systems, IEEE Trans. Power Syst. 3 (3) (1988) 978-985.

[8] S.R. Sanders, J.M. Noworolski, X.Z. Liu, G.C. Verghese, Generalized averaging method for power conversion circuits, IEEE Trans. Power Electron. 6 (2) (1991) 251-259.

[9] J. Freytes, Small-Signal Stability Analysis of Modular Multilevel Converters and Application to MMC-Based Multi-Terminal DC Grids, Ecole Centrale de Lille, 2017 Ph.D. thesis. 